\title{
Contrast sensitivity in multiple sclerosis measured by Cambridge Low Contrast Gratings: a useful clinical test?
}

\author{
J FAHY, D GLYNN, M HUTCHINSON \\ From the Department of Neurology, Adelaide Hospital, Dublin, Ireland
}

SUMmary The Cambridge Low Contrast Gratings (CLCG) were used to assess contrast sensitivity (CS) in 39 patients with clinically definite multiple sclerosis and 60 control subjects. CS was determined in both horizontal and vertical orientations and compared with visual evoked responses (VERs) in the same populations. Only $33 \%$ of patients had abnormal CS whereas $82 \%$ had abnormal VERs. There was no correlation between the degree of abnormalities in CS and VERs. There was no significant difference between CS determined in the vertical and horizontal orientation; however, examination in the vertical orientation increased the number of abnormalities detected by $2(5 \%)$. We conclude that although CS, using the CLCG, is abnormal in a proportion of multiple sclerosis patients its detection is not clinically useful and is insensitive as a measure of subclinical optic neuropathy in multiple sclerosis.

Contrast sensitivity (CS) is defined as the threshold contrast at which a faint pattern of stripes is just visible. Impairment of CS has been demonstrated in a variety of disorders associated with visual loss. CS testing unmasks "hidden visual loss" in multiple sclerosis' and its introduction as a simple and rapid screening test for subclinical optic neuropathy in multiple sclerosis has been proposed. ${ }^{2}$

Tests of CS have evolved from gratings generated on an oscilloscope screen to the recently introduced Cambridge Low Contrast Gratings (CLCG). ${ }^{3}$ The Cambridge gratings measure CS at only one spatial frequency (4 cycles/degree) but when sensitivity to other spatial frequencies is impaired sensitivity at 4 cycles/degree is usually also affected. This test has the advantage of being cheap, easy to use and easy to interpret.

The present paper reports on the use of CLCG as a possible diagnostic test in patients with clinically definite multiple sclerosis. We have also examined two different orientations, horizontal and vertical, in a control and a multiple sclerosis population.

Address for reprint requests: Dr $M$ Hutchinson, Department of Neurology, Adelaide Hospital, Dublin 8, Ireland.

Received 11 October 1988 and in revised form 16 January 1989. Accepted 20 January 1989

\section{Methods}

Contrast Sensitivity Testing

CS was assessed using the CLCG in accordance withe instructions. ${ }^{3}$ The plates were mounted in two orientations, horizontal and vertical. A series of pairs of plates was presented in ascending order of difficulty, a total of 4 times for each eye. Each eye was scored separately, the score reflecting the plate at which the error occurred. Using the conversion table the maximum possible normal score was 560 and the score for each subject was taken as that of the worse eye. The orientation presentation of the plates was randomised.

Visual Evoked Responses (VERs)

VERs were determined using a previously described method. ${ }^{4}$

\section{Subjects}

Thirty nine patients ( 27 female and 12 male) with clinically definite multiple sclerosis ${ }^{5}$ and normal visual acuity $(6 / 6$ Snellen), corrected if necessary, were selected from the multiple sclerosis clinic. The 60 control subjects were hospital staff, students and patients free of neurological and ophthalmologic disease. There were 60 control subjects divided in three age groups, 20-30, 31-50, 51-70 years.

\section{Results}

Control group: The mean scores for CS in each 
orientation for the three age groups are shown in table 1.

The lower limit of normal was taken as mean -2 SD. There was no significant difference between mean scores in the horizontal and vertical orientation in any age group. A decline in CS with age was noted.

Study group: In the multiple sclerosis group 13 patients $(33 \%)$ had abnormal CS whereas 32 patients $(82 \%)$ had abnormal VERs (table 2). Four patients $(10 \%)$ had abnormal CS but normal VERs and they were all less than $\mathbf{4 0}$ years of age.

Inclusion of the vertical orientation increased the number of abnormal CS scores by two (5\%); if the horizontal orientation alone had been assessed 11 (28\%) abnormal CS scores would have been detected. There was no correlation between the degree of abnormality in CS scores and prolongation of VERs.

With increasing age, difference in CS between the multiple sclerosis and control populations becomes less distinct and over 50 years of age the mean scores were similar. Only two abnormal CS scores were recorded in the 16 multiple sclerosis patients older than $40(12$ (75\%) abnormal VERs). Thus $85 \%$ of abnormal CS scores were recorded in the multiple sclerosis patients under 40 years and $11(46 \%)$ of that population had CS and $20(83 \%)$ VER abnormalities.

\section{Discussion}

In this study CS was impaired in only $33 \%$ of a population of clinically definite multiple sclerosis patients. Della Sala et al recently studied 48 multiple sclerosis patients using a similar test ${ }^{2}$ and found that $73 \%$ had abnormal CS. In both studies the incidence of VER abnormality was similar. The large discrepancy in CS abnormalities reported may be due to differences in examination methods and the groups studied. Our multiple sclerosis population consisted of clinically definite patients from a multiple sclerosis clinic, the only other criterion being normal visual acuity. The Italian study group had a high incidence of subjective ophthalmic complaints which may relate to the greater frequency of CS abnormalities found. The age structure in both studies was similar.

Table 1 The mean scores and standard deviation (SD) for contrast sensitivity for the three age groups of the control population

\begin{tabular}{lll}
\hline & \multicolumn{2}{l}{ CS score $(S D)$} \\
\cline { 2 - 3 } Age groups & Vertical & Horizontal \\
\hline $20-30$ & $440(115)$ & $400(95)$ \\
$31-50$ & $310(70)$ & $270(65)$ \\
$51-70$ & $210(20)$ & $230(30)$ \\
\hline
\end{tabular}

Table 2 The frequency of abnormal contrast sensitivity (CS) and visually evoked responses (VER) in the multiple sclerosis patients by age group

\begin{tabular}{llrl}
\hline & \multicolumn{3}{l}{ Age groups } \\
\cline { 2 - 4 } & $20-30$ & $31-50$ & $51-70$ \\
\hline CS Abnormal & $4 / 10$ & $9 / 23$ & $0 / 6$ \\
VER Abnormal & $9 / 10$ & $17 / 23$ & $6 / 6$ \\
Both Abnormal & $3 / 10$ & $7 / 23$ & $0 / 6$ \\
CS only Abnormal & $1 / 10$ & $3 / 23$ & $0 / 6$ \\
VER only Abnormal & $6 / 10$ & $10 / 23$ & $6 / 6$ \\
\hline
\end{tabular}

In this study there was a $5 \%$ increase in abnormal CS scores by testing in two different orientations and previous reports of spatial orientation in VER testing have recorded a similar phenomenon. ${ }^{6}$ Thus CS is orientation sensitive and testing in different orientations can increase the sensitivity of the test.

The decline in CS with age in the control population and the approximation of means in the control and multiple sclerosis populations with increasing age renders CS testing over the age of $\mathbf{4 0}$ unproductive (only $13 \%$ of our multiple sclerosis population over 40 years had abnormal CS).

The main object of this study was to determine whether CS testing using the CLCG was a useful clinical tool. The test is easy to perform, to interpret and examination in two orientations takes no longer than 10 minutes. The luminance requirements limit its use as a bedside or clinic test. We feel that the CLCG test of CS is unlikely to become a useful test of subclinical optic neuropathy in multiple sclerosis because of its low sensitivity, the requirement of normal visual acuity, age related decline in CS and the necessity to have a specially standardised area for its use.

\section{References}

1 Regan D, Silver R, Murray TJ. Visual acuity and contrast sensitivity in multiple sclerosis: hidden visual loss. Brain 1977;100:563-79.

2 Della Sala S, Comi G, Martinelli V, Somazzi L, Wilkins AJ. The rapid assessment of visual dysfunction in multiple sclerosis. J Neurol Neurosurg Psychiatry 1987;50:840-6.

3 Cambridge Low Contrast Gratings. London: Clement Clarke International Ltd, 1987.

4 Hutchinson M, Martin EA, Maguire P, Glynn D, Mansfield M, Feighery $C$. Visual evoked responses and immunoglobulin abnormalities in the diagnosis of multiple sclerosis. Acta Neurol Scand 1983;68:90-5.

5 McDonald WI, Halliday AM. Diagnosis and classification of multiple sclerosis. Br Med Bull 1977;33:4-8.

6 Coupland SG, Kirkham TH. Orientation-specific visual evoked potential deficits in multiple sclerosis. Can J Neurol Sci 1982;9:331-7. 